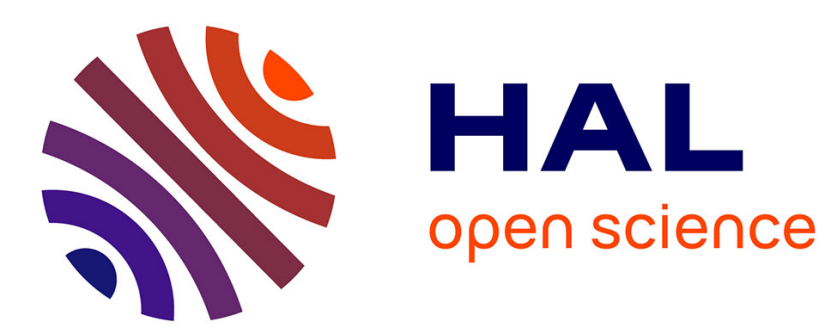

\title{
Control of an airborne wind energy system with a Magnus effect
}

\author{
Ahmad Hably, Jonathan Dumon, Garrett Smith
}

\section{To cite this version:}

Ahmad Hably, Jonathan Dumon, Garrett Smith. Control of an airborne wind energy system with a Magnus effect. ACC 2016 - American Control Conference, Jul 2016, Boston, MA, United States. hal-01272253

\section{HAL Id: hal-01272253 \\ https://hal.science/hal-01272253}

Submitted on 10 Feb 2016

HAL is a multi-disciplinary open access archive for the deposit and dissemination of scientific research documents, whether they are published or not. The documents may come from teaching and research institutions in France or abroad, or from public or private research centers.
L'archive ouverte pluridisciplinaire HAL, est destinée au dépôt et à la diffusion de documents scientifiques de niveau recherche, publiés ou non, émanant des établissements d'enseignement et de recherche français ou étrangers, des laboratoires publics ou privés. 


\section{Control of an airborne wind energy system with a Magnus effect}

\author{
Ahmad Hably*, Jonathan Dumon \\ Univ. Grenoble Alpes, GIPSA-Lab, \\ F-38000 Grenoble, France. \\ \{ahmad.hably, jonathan.dumon\}@gipsa-lab.fr
}

\author{
Garrett Smith \\ Wind Fisher S.A.S., 2 allee du Vivarais, \\ 31770 Colomiers, France. \\ garrett.smith@wind-fisher.com
}

\begin{abstract}
The control of an airborne wind energy system with a Magnus effect device has been studied. The proposed strategy aims to control the amount of the output energy produced for a given cycle. The control scheme has been applied numerically and experimentally on a small scale indoor setup of Gipsa-lab, and validated numerically for Omnidea's experimental platform. Results have shown the good performance of the proposed control strategy.
\end{abstract}

\section{INTRODUCTION}

Airborne wind energy systems have attracted a lot of interest in the last few years. Due to the quality of higher altitude wind which is stronger and more persistent they are considered as a promising alternative to traditional wind turbines with limitations related to weight and size, and therefore the investment cost when the targeted power increases. There are many ways to capture the wind energy using aerodynamic surfaces with moving center of mass and orientation. The existing prototypes can be divided into two main classes:

- On-ground production using the lift mode as noted in [1]. A traction phase, in which the airfoil is pulled by the wind, unrolling the cable which turns a groundbased electrical machine; and a recovery phase, that begins when the cable reaches its predefined maximum length, and hence needs to be reeled-in, an operation that consumes energy. These systems are studied by [2], Kitegen [3], and Ampyx Power [4].

- On-board production using the drag mode. The generator is embedded and electric energy is produced in the sky and sent to the ground using conducting cables. This type of system is investigated for example by Makani Power [5].

Most of the aforementioned systems use either flexible kites or rigid wings. However, Omnidea Lda has proposed to use the Magnus effect in its HAWE project [7]. The operation principle of their platform is based on the rotation of a buoyant cylinder attached to the ground by cables. The Magnus effect generates an aerodynamic lift force which depends on the apparent wind speed with relation to the cylinder and the cylinder rotational speed itself. Electrical energy is produced using the pumping mode. The Magnus cylinder is almost static and therefore the apparent wind speed remains close to the real wind speed [8]. This type of system using the Magnus effect must be explored. In addition, a key difference compared to the AWE systems using kites or wings whose lift and drag coefficients depend on the angle of attack, is that Magnus effect systems are independent of angle of attack. The control of the angle of attack is sometimes critical and depends on some parameters which are difficult to measure.

The paper is organized as follows. Section II introduces the system modeling. The control strategy is presented in Sect.III. This control strategy is applied to the experimental platform described in Sect.IV. Both simulation and experimental results are shown in Sect.V. A numerical application of the proposed control strategy for Omnidea' s experimental platform is presented in Sect.VI. Section VII provides some conclusions and perspectives.

\section{THE SYSTEM MODELING}

The airborne wind energy system under study is composed of a ground-based motor that will supply a traction force to a tether connected to a light-weight rotating Magnus cylinder. In this study the movement of the Magnus cylinder is limited to the vertical plane. The system's dynamical model can be given by:

$$
\begin{aligned}
\ddot{\theta} & =\frac{1}{r}\left[-2 \dot{\theta} \dot{r}+\frac{F_{T}}{M_{M a g}}\right] \\
\ddot{r} & =\frac{1}{M_{M a g}+M_{D}}\left[r \dot{\theta}^{2} M_{M a g}+F_{R}-T\right] \\
\dot{T} & =\beta_{T}\left(u_{T}-T\right)
\end{aligned}
$$

where $r$ is the tether length from the Magnus cylinder to the motor on the ground, $\theta$ is the angle that the tether makes with respect to the horizon, $M_{D}=\frac{I}{R_{d}^{2}}$ with $I$ the inertia of the ground-based motor and $R_{d}$ its radius, $T$ is the traction on the tether. The Magnus cylinder mass $M_{\mathrm{Mag}}$ is the sum of cylinder mass $M$, the mass of the gas used to fill it, and $M_{l}$ :

$$
M_{\mathrm{Mag}}=M+V_{o} \rho_{\mathrm{gas}}+M_{l} r
$$

with $\rho_{\text {gas }}$ is the gas density and $M_{l}$ denotes the mass per tether length and $V_{o}$ is the volume of the Magnus cylinder. Equation 3 represents the first order dynamic response of the actuator used in the experimental platform to control the traction force in the tether. $F_{R}$ and $F_{T}$ are respectively the radial and tangential forces acting on the Magnus cylinder due to lift force $L$, drag force $D$, the weight of the Magnus cylinder $P$, and the buoyant force $B$. Denoting $s_{i}:=\sin \left(\theta-\alpha_{w}\right)$ and $c_{i}:=\cos \left(\theta-\alpha_{w}\right)$, these forces can be expressed as follows:

$$
\begin{aligned}
& F_{R}=-T+L s_{i}+D c_{i}-P \sin \theta+B \sin \theta \\
& F_{T}=L c_{i}-D s_{i}-P \cos \theta+B \cos \theta
\end{aligned}
$$




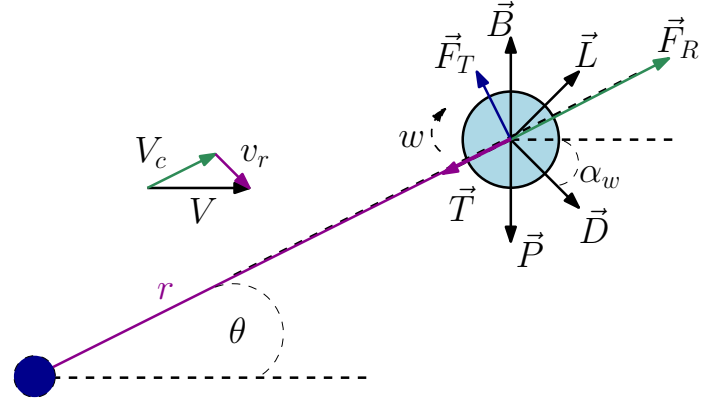

Fig. 1. The forces acting on the Magnus cylinder airborne wind energy system.

with $\alpha_{w}$ is the wind angle defined later in equation (11). Lift and drag forces can be expressed by:

$$
L=0.5 \rho S v_{r}^{2} C_{L}, \quad D=0.5 \rho S v_{r}^{2} C_{D}
$$

where $\rho$ is the air density, $S$ is the Magnus cylinder projected surface area, $v_{r}$ as we will see later is the norm of the apparent wind velocity vector (equation 12). The buoyancy force can be calculated from the Archmiedes's principle:

$$
B=\rho V_{o} g
$$

Vertical relative airspeed $v_{v}$ and horizontal relative airspeed $v_{h}$ depend on the motion of the rotating Magnus cylinder. One has:

$$
\begin{aligned}
& v_{h}=V+r \dot{\theta} \sin \theta-\dot{r} \cos \theta \\
& v_{v}=-(r \dot{\theta} \cos \theta+\dot{r} \sin \theta)
\end{aligned}
$$

with $V$ is the airspeed with respect to the ground. Using these equations, one obtains, $v_{r}$, the norm of the apparent wind velocity vector and the angle of this vector with respect to the ground $\alpha_{w}$.

$$
\begin{aligned}
\alpha_{w} & =\arctan \frac{-(r \dot{\theta} \cos \theta+\dot{r} \sin \theta)}{V+r \dot{\theta} \sin \theta-\dot{r} \cos \theta} \\
v_{r} & =\sqrt{(r \dot{\theta} \cos \theta+\dot{r} \sin \theta)^{2}+(V+r \dot{\theta} \sin \theta-\dot{r} \cos \theta)^{2}}
\end{aligned}
$$

For lifting devices using the Magnus effect, aerodynamic lift coefficient $C_{L}$ and drag coefficient $C_{D}$ are functions of the spin ratio $X$ and not of the angle of attack as for airfoil wings. The Magnus cylinder spin ratio is given by the following equation [8]:

$$
X=\frac{w R}{v_{r}}
$$

with $w$ is the Magnus cylinder rotational velocity and $R$ is its radius.

\section{THE CONTROL STRATEGY}

The control strategy to be applied on the Magnus-based system aims to control the amount of energy produced by forcing some variables, namely the tether traction force $T$ and its length $r$, to track some "optimized" profile related to desired power. This system has two phases: a generation (traction) phase where the tether is pulled by the Magnus cylinder using the aerodynamic forces and then a consumption (recovery) phase where the Magnus cylinder is pulled by the tether to return to its initial departure point in order to start a new cycle. For simplicity, desired power $P_{\text {ref }}$ will be assumed constant, but the control strategy can be adapted to varying $P_{r e f}$ as we will see in the results section. During the cycle, the Magnus cylinder moves from minimum position $r_{\min }$ to a maximum position $r_{\max }$ at a speed $\dot{r}_{\text {prod }}$ and $\dot{r}_{r e c}$ respectively during production and recovery phases. The proposed algorithm is based on the following rules. A given cycle is defined by the beginning of the recovery phase $\left(t_{0}\right)$ until the end of the production phase $\left(t_{1}\right)$.

- The consumed energy is measured from $t_{0}$ to time $t$

$$
E_{\text {rec }}(t)=\int_{t_{0}}^{t} P_{m e s} d t
$$

$P_{m e s}$ is the measured power defined by:

$$
P_{m e s}=P_{g e n}+P_{M a g}
$$

with $P_{\text {gen }}$ is the measured power produced or consumed by the generator on the ground and $P_{M a g}$ is the power consumed by the Magnus actuator.

- At the end of the recovery cycle, the remaining energy to be produced $E_{\text {prod }}$ has to satisfy $E\left(t_{1}\right)=P_{\text {ref }} \times$ $\left(t_{1}-t_{0}\right)=E_{\text {ref }}$.

$$
E_{\text {prod }}(t)=E_{\text {ref }}-E_{\text {rec }}(t)
$$

- The traction force has to satisfy

$$
T_{\text {ref }}=\frac{1}{\dot{r}_{\text {prod }}} \frac{E_{\text {ref }}-E_{\text {rec }}(t)}{\left(t_{1}-t\right)}
$$

Three controllers have been used to implement this control strategy (2). Controller $K_{3}$ is used to find the desired tension $T_{\text {ref }}$ as a function of the desired power $P_{\text {ref }}$ (Eq. 17). This desired tension is controlled in its turn by a controller, denoted by $K_{2}$, to get the Magnus cylinder spin ration $X$. The tether length is controlled by $K_{1}$ in order to obtain the traction control $u_{T}$. During the cycle, the Magnus cylinder moves from $r_{\text {min }}$ to $r_{\text {max }}$ at a speed $\dot{r}_{\text {prod }}$ and $\dot{r}_{r e c}$ respectively during production and recovery phases. The parameters $K_{1}$ and $K_{2}$

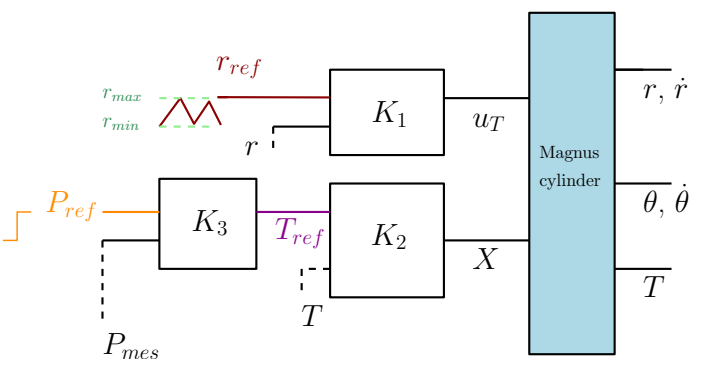

Fig. 2. An overview of the proposed control system.

are tuned empirically to separate the dynamics of the inner 
and outer loop and in order to have a fast response time to obtain maximum power for a given wind speed as we will see later in the results section.

\section{PLATFORM DESCRIPTION}

In order to validate the proposed system and the control strategy, the Gipsa-lab experimental test bench is used. This experimental setup was built for our work using rigid wings [9], the same algorithm was also used in [10]. This indoor experimental setup gives us some flexibility and allows us to test our prototypes and the proposed control strategies sheltered from outside weather conditions. It is composed of a wind tunnel, the Magnus cylinder, and the ground station.

\section{A. Wind Tunnel}

The wind tunnel is composed of 9 brushless motors with 2-blade fans of $0.355 \mathrm{~m}$ diameter. These motors, $800 \mathrm{~W}$ each, are distributed on a surface of $1.85 \mathrm{~m}^{2}$. Turbulent air flow is produced at speeds up to $9 \mathrm{~m} / \mathrm{s}$. A hot wire wind speed sensor ( 1 measurement per second with a serial interface) is used to measure the airspeed. Controllers are implemented on the experimental setup using the xPC target real-time toolbox of Matlab (Fig. 3).

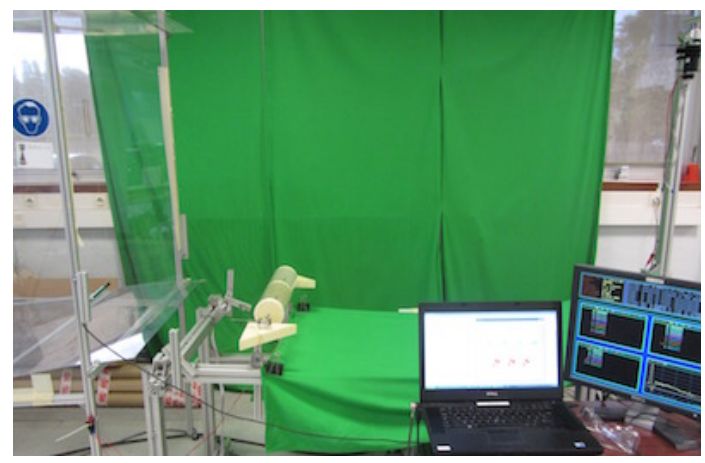

Fig. 3. The development and target computers for real-time experiments using Matlab/Simulink xPC target real-time toolbox.

\section{B. The Magnus Cylinder}

The Magnus cylinder used in our platform is a light-weight cylinder built with carbon rods, polystyrene and transparent plastic (Fig. 4). The rotation of the Magnus cylinder is provided by one mini DC motor mounted at one extremity of the Magnus cylinder. Its current control and speed sensing is done using a homemade driver. The parameters of the Magnus cylinder are given in Table I.

\section{The Ground Station}

The ground station is composed of dynamo-motor system Maxon 2260L DC $100 \mathrm{~W}$ driven by a 4 quadrants amplifier Maxon ADS 50/10. Two incremental encoders provide measurement of the angle $\theta$ and the tether length $r$. Control references of DC motors are sent to drivers with a DAC PCI DA S1200 from Measurement Computing and a torque sensor provides an accurate measurement of tether tension.

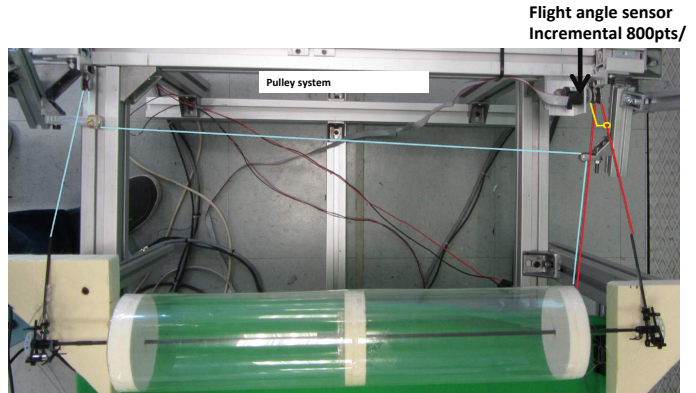

Fig. 4. The Magnus cyinder.

TABLE I

PARAMETERS OF THE MAGNUS CYLINDER

\begin{tabular}{lll}
\hline Symbol & Name & Value \\
\hline$M_{\text {Mag }}$ & Magnus cylinder mass & $0.11 \mathrm{Kg}$ \\
$M_{l}$ & Mass per tether length & $0 \mathrm{Kg} / \mathrm{m}$ (neglected) \\
$R$ & Magnus cylinder radius & $0.047 \mathrm{~m}$ \\
$L_{m}$ & Magnus cylinder length & $0.45 \mathrm{~m}$ \\
$M_{I M}$ & Rotor mass & $0.0481 \mathrm{Kg}$ \\
$\rho$ & Air density & $1.225 \mathrm{Kg} / \mathrm{m}^{3}$ \\
\hline
\end{tabular}

\section{RESULTS}

Before applying the control strategy presented previously, an important phase of characterizing the different elements of the experimental setup was needed.

\section{A. Characterization}

Firstly, the response time of the DC motor used to rotate the Magnus cylinder is characterized and its energy consumption is quantified for different wind speeds. The second step is to identify the lift and drag coefficients as a function of the spin ratio. The results are very near to the theoretical results [11] used in [12] where the drag and lift aerodynamic coefficients are calculated as a function of speed ratio $X$.

$$
\begin{aligned}
& C_{D}=0.73 X^{2}-1.2 X+1.2131 \\
& C_{L}=0.0126 X^{4}-0.2004 X^{3}+0.7482 X^{2}+1.3447 X-0.2
\end{aligned}
$$

The last step in the characterization phase is to find the limits of our platform. We have noticed that friction in the pulleys is significant. We have measured the tension in the tether as a function of the tether length $r$ for different rotational speed $w$ of the Magnus cylinder and the tether speed $\dot{r}$ and we have found our platform can provide a limited difference of traction force that can be used to produce energy. This is shown in the difference between the upper and lower zones of Fig. 6

\section{B. Simulation Results}

In this section, the proposed control strategy is tested numerically. Our objective is to validate the control strategy and to have cycle with a positive production result. For this, the controller block $K_{3}$ is not active in these tests because of 


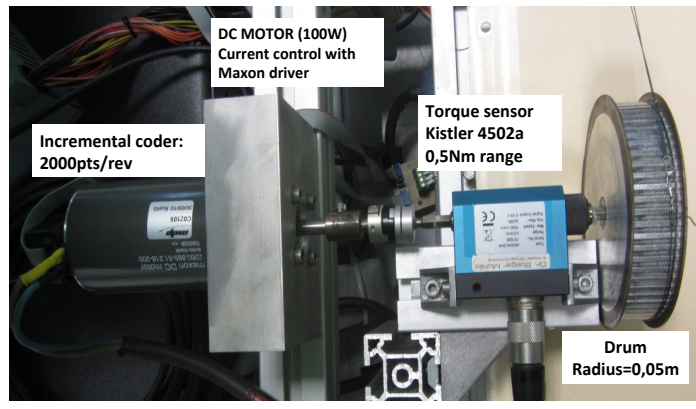

Fig. 5. The ground station.

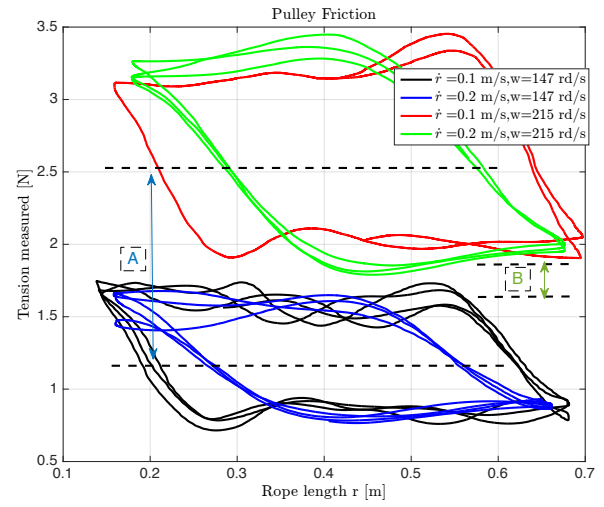

Fig. 6. The measured tension as a function of tether speed $\dot{r}$ (rp in the figure) and the Magnus cylinder rotaltional speed $w$. The zone $\mathbf{A}$ is the possible force difference that can be used to produce energy. This zone is reduced to zone $\mathbf{B}$ due to the pulleys' friction.

the identified friction in the setup. The following conditions are used.

- The minimum tether length $r_{\min }=0.1 \mathrm{~m}$ and its maximum is $r_{\max }=0.7 \mathrm{~m}$. These limits are imposed by the wind window of the wind tunnel.

- The tether speed in the traction phase $\dot{r}_{\text {prod }}=0.1 \mathrm{~m} / \mathrm{s}$ and in the recovery one $\dot{r}_{r e c}=-0.1 \mathrm{~m} / \mathrm{s}$.

- The response time of the actuators used to turn the Magnus cylinder is too long in order to maintain a constant value of $X$. In addition, one has fast variations of the relative wind speed related to oscillation on $\theta$. For this and in order to validate our control strategy, we have chosen to control only $w$ which gives a mean $X$ value. A constant rotational Magnus cylinder speed is used in the traction phase $w_{\text {prod }}=200 \mathrm{rd} / \mathrm{s}$ and and in the recovery phase $w_{r e c}=140 \mathrm{rd} / \mathrm{s}$. This corresponds by approximating $v_{r} \approx V$ to have a spin ratio in the traction phase $X_{\text {prod }}=1.5161$ and $X_{r e c}=1.0613$ in the recovery phase.

- The wind speed is $6.2 \mathrm{~m} / \mathrm{s}$. Reynolds number is $4 \times 10^{4}$. The tether length follows perfectly the desired position as shown on Fig. 7. As expected, the traction force increases as the rotational Magnus cylinder' speed increases. The application of this control strategy enables us to produce a positive result as shown on Fig. 8 .
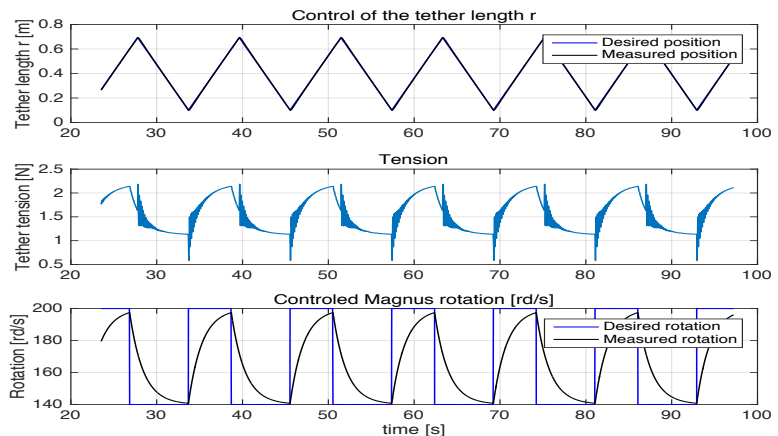

Fig. 7. Tether length, tether tension and the Magnus rotational speed as function of time in the simulation of small scale system. The oscillation in the tether tension is due to the choice of controller parameters.

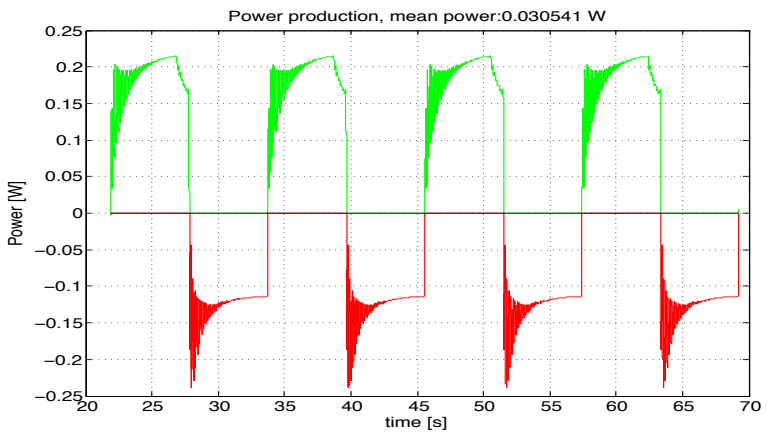

Fig. 8. The power produced in the simulation.

\section{Experimental Results}

The same control strategy has been experimentally applied to the setup using the same conditions used in the previous section. Similar results have been obtained as shown in the Figs. 9-11. A movie that shows the experimental results can be found on our website [13].

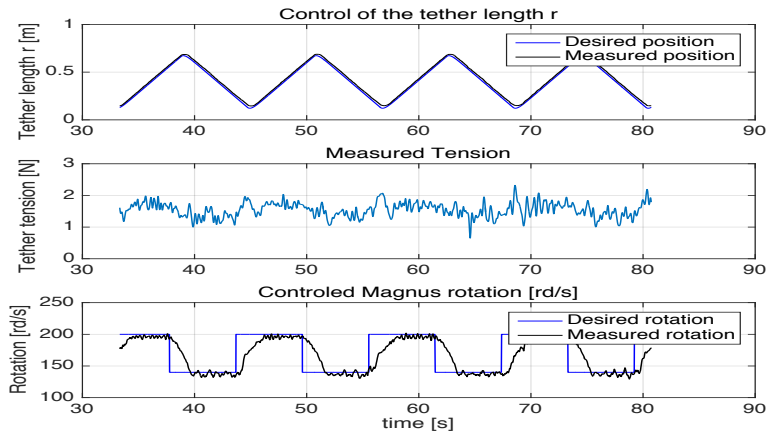

Fig. 9. Tether length, tether tension and the Magnus rotational speed as function of time in the experimentation on Gipsa-lab platform. 


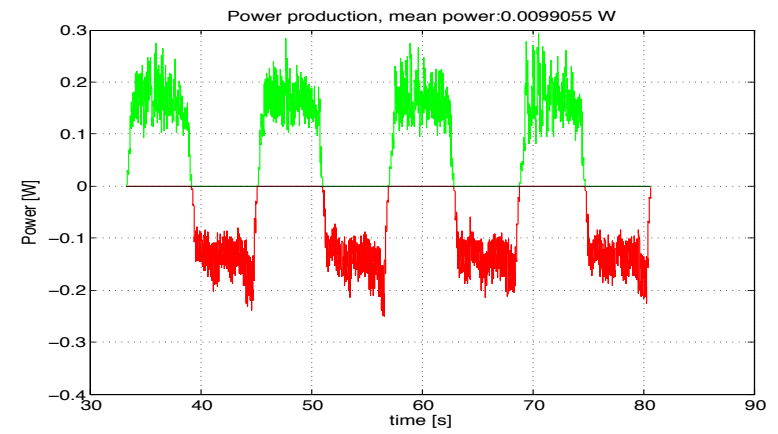

Fig. 10. The power produced in the experimentation on Gipsa-lab platform.

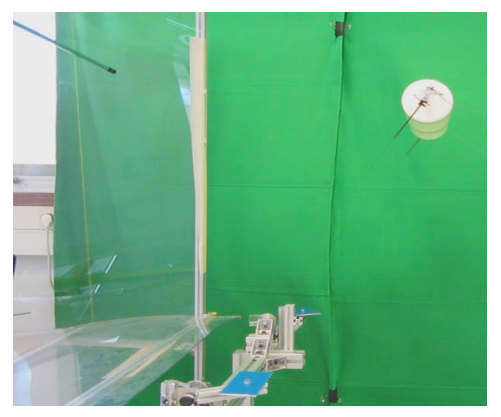

Fig. 11. The Magnus cylinder airborne.

\section{NUMERICAL APPLICATION TO OMNIDEA SYSTEM}

The complete control strategy has been numerically applied for Omnidea's platform. We have taken the dimensions of the Magnus cylinder currently in service. Its parameters are listed in Table II. The Magnus lift and drag coefficients used for

TABLE II

PARAMETERS OF OMNIDEA MAGNUS CYLINDER

\begin{tabular}{lll}
\hline Symbol & Name & Value \\
\hline$M_{\mathrm{Mag}}$ & Magnus cylinder mass & $91.22 \mathrm{Kg}$ \\
$R$ & Magnus cylinder radius & $1.25 \mathrm{~m}$ \\
$L_{m}$ & Magnus cylinder length & $16 \mathrm{~m}$ \\
$\rho_{H e}$ & Helium density & $0.1427 \mathrm{Kg} / \mathrm{m}^{3}$ \\
$\rho_{\text {air }}$ & Air density & $1.225 \mathrm{Kg} / \mathrm{m}^{3}$ \\
$M_{l}$ & Mass per tether length & $0.2 \mathrm{Kg} / \mathrm{m}$ \\
$M_{D}$ & Ground station rotor mass & $2000 \mathrm{Kg}$ \\
\hline
\end{tabular}

Reynolds number $R_{e}=3.8 \times 10^{4}$ :

$$
\begin{aligned}
& C_{D}=-0.0211 X^{3}+0.1837 X^{2}+0.1183 X+0.5 \\
& C_{L}=0.0126 X^{4}-0.2004 X^{3}+0.7482 X^{2}+1.3447 X
\end{aligned}
$$

We want to have an optimal production cycle with vertical trajectories similar to those suggested in [12]. We have determined the feasibility regions for $r_{\min }=200 \mathrm{~m}$ and $r_{\max }=300 \mathrm{~m}$. For a wind speed $V=10 \mathrm{~m} / \mathrm{s}$, the tether speed in the traction phase $\dot{r}_{\text {prod }}$ and in the recovery one $\dot{r}_{r e c}$ are found numerically offline. One gets $\dot{r}_{\text {prod }}=0.33 \times V$

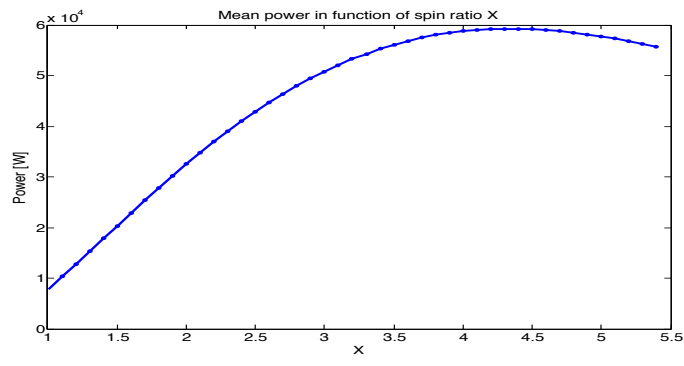

Fig. 12. Omnidea results: The variation of the mean power as a function of the spin ratio $X$.

and $\dot{r}_{r e c}=-0.52 \times V$. The choice of the spin ratio value of Omnidea's Magnus cylinder depends on the following objectives: 1) To maximize the lift-to-drag ratio $\frac{L}{D}$, one has to take $X=2.4$, and 2) To maximize the aerodynamic forces, i.e $\sqrt{C_{L}^{2}+C_{D}^{2}}$, the spin ratio must be equal to $X=5.4$.

For a vertical trajectory, the spin ratio will take a value between $X=5.4$, which maximizes power without taking part of the relative wind, and $X=3.6$, which maximizes the crosswind power if one reaches the theoretical relative speed $v r=\frac{L}{D} V$. By simulating this system at a wind speed $V=10$ $\mathrm{m} / \mathrm{s}$ (see next section for details), we get the mean power produced during a full cycle as a function of $X$ (Fig. 12). Note that we do not consider here the motor consumption that actuates the Magnus cylinder. The maximum power is $P_{\text {moy }}=59.23 \mathrm{KW}$ for $X=4.3$ which is between 3.6 and 5.4 as expected. For this set of parameters, the energetic performance is $1.48 \mathrm{~kW} / \mathrm{m}^{2}$ which is consistent with 1.25 $\mathrm{kW} / \mathrm{m}^{2}$ found in [12].

\section{A. Nominal production cycle}

In this section, the results of the production cycle are presented. In order to have a smooth movement of the Magnus cylinder, the reference tether length $r_{r e f}$ is filtered by $\frac{1}{\left(\tau_{R} s+1\right)^{2}}$ with $\tau_{R}=2 \mathrm{~s}$. The PID controller $K_{1}$ parameters are $K_{p}=8250 \mathrm{~N} / \mathrm{m}, K_{i}=1.32 \mathrm{~N} /(\mathrm{m} . \mathrm{s}), K_{d}=45 \times 10^{3} \mathrm{~N} . \mathrm{s} / \mathrm{m}$. We find that the relative wind speed increases thanks to the evolution of flight angle $\theta$ which produces the vertical shape of the cycle (Fig. 13). with a maximum of $v_{r}=14.26 \mathrm{~m} / \mathrm{s}$ in the production phase and $v_{r}=-14.79 \mathrm{~m} / \mathrm{s}$ in the recovery phase. On Fig. 14, we show the evolution of the control. One can find the maximum tension in the tether is $T_{\max }=42.4$ $\mathrm{kN}$, the maximum rotational speed $w_{\max }=49.02 \mathrm{rd} / \mathrm{s}$. The production speed is $3.3 \mathrm{~m} / \mathrm{s}$ with an overshoot measured at $8 \mathrm{~m} / \mathrm{s}$, the traction speed is set to $-5.2 \mathrm{~m} / \mathrm{s}$, without any observed overshoot. Omnidea' current system cannot completely meet these values since the announced maximum force is $5 \mathrm{kN}$ with a maximum rotational speed of $9.42 \mathrm{rd} / \mathrm{s}$.

\section{B. Energy control}

A production cycle using the complete control strategy has been tested. To find the control parameters of the controller $K_{2}$ (PD controller), we have chosen the increasing line 


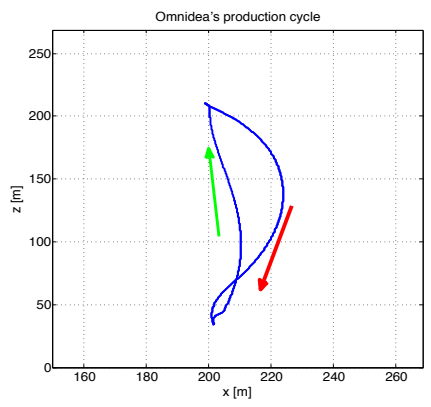

Fig. 13. The production cycles of Omnidea's platform. The direction of the arrows indicates the movment of the Magnus cylinder: Green for the traction phase and red for the recovery one.

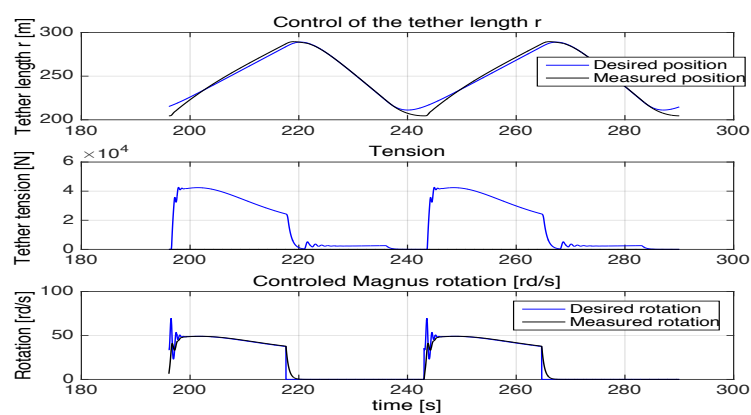

Fig. 14. Tether length, tether tension and the Magnus rotational speed as function of time for Omnidea's platform.

slope of Fig. 12 between $X=1$ and $X=4.3$. The control parameters are then $K_{p}=6.4 \times 10^{-3} N^{-1}$ and $K_{d}=6.4 \times 10^{-3} \mathrm{~s} / \mathrm{N}$. One can clearly see the performance of the proposed control strategy (Fig. 15). The measure produced power will follow the desired one even in the presence of noise on the wind speed. The control variables are shown on Fig. 16. It is worth noting that if the output of PD is saturated, one can simply apply a very large reference to achieve the maximum power, with $\mathrm{X}=4.3$ throughout the production phase
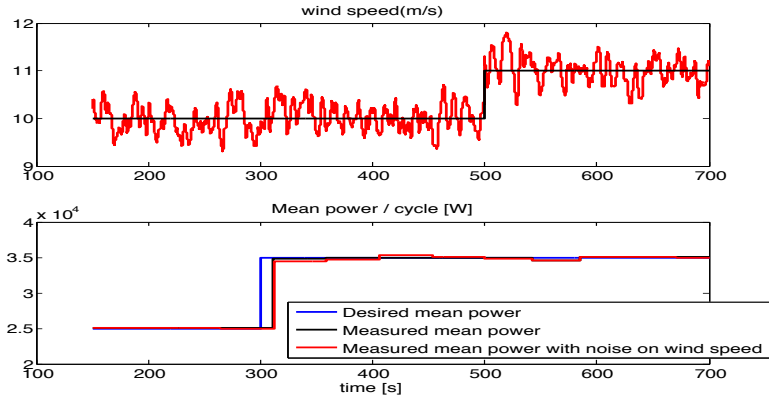

Fig. 15. The mean power produced as function of the desired power and change in wind speed for Omnidea's platform. Noise is added to the wind speed to test the performance of the control strategy.

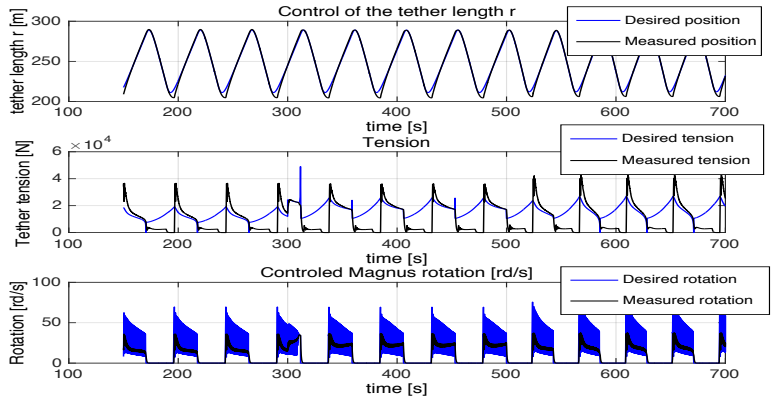

Fig. 16. The evolution of the control variables in absence of noise for Omnidea's platform.

\section{CONCLUSIONS AND PERSPECTIVES}

In this paper, we have presented the control of airborne wind energy system based on a Magnus cylinder. The indoor small-scale experiments have enabled us to master different aspects of the system and to validate part of our approach. The Magnus model was validated for a spin ratio ranging from 1 to approximately 2.3 . Our goal for future work is experiment with models capable of spin ratio greater than 5.5. The small size of our wind tunnel does not allow us to reach tethers speeds that would achieve the simulated performance $1.48 \mathrm{~kW} / \mathrm{m}^{2}$, but faster dynamics of the actuators rotating the Magnus cylinder would allows us to achieve more vertical cycles thus experiencing the dynamic exploitation of the relative wind due to the increase of the flight angle $\theta$.

\section{ACKNOWLEDGMENT}

The authors of this paper would like to thank the technical staff of Gipsa-lab and the trainees Azzam Alwann, Alexandre Kajiyama and Pierre Estadieu.

\section{REFERENCES}

[1] M. Loyd, "Crosswind kite power," J. ENERGY, vol. 4(3), 1980.

[2] L. Fagiano, M. Milanese, and D. Piga, "High-altitude wind power generation," Energy Conversion, IEEE Transactions on, vol. 25, no. 1, pp. 168-180, 2010.

[3] M. Canale, L.Fagiano, and M.Milanese, "Kitegen: A revolution in wind energy generation," Energy, vol. 34, pp. 355-361, 2009.

[4] "http://www.ampyxpower.com."

[5] http://www.makanipower.com/.

[6] "http://www.altaerosenergies.com."

[7] "http://www.omnidea.net/hawe/."

[8] J. Seifert, "A review of the magnus effect in aeronautics," Progress in Aerospace Sciences, vol. 55, pp. 17-45, 2012.

[9] A. Hably, R. Lozano, Alamir, and J. M. Dumon, "Observer-based control of a tethered wing wind power system : indoor real-time experiment," in The 2013 American Control Conference, (ACC2013)., 2013.

[10] R. Lozano, J. Dumon, A. Hably, and M. Alamir, "Energy production control of an experimental kite system in presence of wind gusts," in Intelligent Robots and Systems (IROS), 2013 IEEE/RSJ International Conference on. IEEE, 2013, pp. 2452-2459.

[11] F. White, Fluid mechanics. McGraw-Hill Higher Education, 2015.

[12] M. Milutinović, M. Čorić, and J. Deur, "Operating cycle optimization for a magnus effect-based airborne wind energy system," Energy conversion and management, vol. 90, pp. 154-165, 2015.

[13] "http://www.gipsa-lab.grenoble-inp.fr/recherche/platesformes.php?id_plateforme=70."

[14] “http://omnidea.net/site/index.php." 